Pacific Journal of Mathematics

INVOLUTIONS ON LOCALLY COMPACT RINGS 


\section{INVOLUTIONS ON LOCALLY COMPACT RINGS}

\section{PAUL CIVIN}

By a proper involution * on a ring $R$ we mean a mapping $x \rightarrow x^{*}$ defined on $R$ with the following properties:

(i) $(x+y)^{*}=x^{*}+y^{*}$,

(ii) $(x y)^{*}=y^{*} x^{*}$,

(iii) $\left(x^{*}\right)^{*}=x$ and

(iv) $x x^{*}=0$ if and only if $x=0$. If (iv) is not assumed, the mapping is simply termed an involution. If $F$ is a field with an involution \# and $R$ is an algebra over $F$, we say that an involution on $R$ is an algebra involution if in addition to (i)-(iv) above the following holds:

(v) $(\alpha x)^{*}=\alpha^{\sharp} x^{*}$ for all $x \in R$ and $\alpha \in F$.

We are concerned principally with involutions on two types of locally compact semi-simple rings, namely those which are compact or connected. The main result is that involutions on such rings are automatically continuous. As a byproduct we determine the form of any proper involution on a total matric ring $R$ over a division ring. If in addition $R$ is topological and the division ring admits only continuous involutions, then we note that $R$ has only continuous involutions.

Lemma Let $D$ be a division ring with center $Z$. Let $R$ be a total matric ring over $D$. Any ring involution * on $R$ induces an involution \# on $Z$, and * is an algebra involution on $R$ with respect to the involution \# on $Z$.

Direct calculation shows that the center of $R$ consists of the totality of elements of the form $\alpha I$ where $\alpha \in Z$ and $I$ is the identity of $R$. Suppose $x$ is in the center of $R$ and $y \in R$, then $x^{*} y=\left(y^{*} x\right)^{*}=\left(x y^{*}\right)^{*}=$ $y x^{*}$, so $x^{*}$ is in the center of $R$. Since $I^{*}=I$ is immediate, it follows that for any $\alpha \in Z$, there is a $\beta \in Z$ such that $(\alpha I)^{*}=\beta I$. Denote $\beta$ by $\alpha^{\#}$. It is clear that \# is an involution on $Z$. Moreover, if $\alpha \in Z$ and $x \in R,(\alpha x)^{*}=[(\alpha I) x]^{*}=x^{*} \alpha^{\sharp} I=\alpha^{\sharp} x$, so * is an algebra involution on $R$ with respect to the involution \# on $Z$.

THEOREM 2. Let $R$ be a total matric ring over $D$, where $D$ is a division ring with center $Z$. Let ${ }^{*}$ be a proper ring involution on $R$, and let \# be the induced involution on $Z$. Then there exist a set of matrix units $\left\{g_{i j}\right\}$ in $R$ such that $g_{i i}^{*}=g_{i i}$ and a set of non-zero elements $\gamma_{i}$ of $Z$ such that $\gamma_{i}^{*}=\gamma_{i}$ such that the involution * has the following form: If $x=\sum \alpha_{i j} e_{i j}$, with $\alpha_{i j} \in D$, then $x^{*}=\sum \gamma_{j}^{-1} \alpha_{i j} \gamma_{i} e_{j i}$.

Received August 31, 1959, and in revised form January 28, 1960. This research was supported in part by the National Science Foundation, under grant NSF-G5865. 
Let $e_{i j}, i, j=1, \cdots, n$ be a set of matric units for $R$. The right ideal $e_{11} R$ is minimal, so by a theorem of Rickart [7] there is a unique idempotent $u_{1} \in e_{11} R$ such that $u_{1}^{*}=u_{1} \neq 0$. Let $L_{1}=R u_{1}$, and $L_{k}=$ $R e_{1 k}=R e_{k k}, k=2, \cdots, n$. The $L_{k}$ are minimal left ideals so by the Rickart theorem there are unique idempotents $u_{k} \in L_{k}$ such that $u_{k}^{*}=$ $u_{k} \neq 0, k=1, \cdots, n$.

We denote by $[A, B, \cdots, C]$ the smallest left ideal containing $A, B$, $\cdots, C$. The linear independence of $u_{1}$ and the $e_{1 k}, k=2, \cdots, n$ implies that $L_{k} \not \subset\left[L_{1}, \cdots, L_{k-1}\right]$ for $1<k \leqq n$. It is readily verified that $R=$ $\left[L_{1}, \cdots, L_{n}\right]$.

Let $g_{1}=u_{1}$ and suppose that $g_{1}, \cdots, g_{k-1}$ have been defined so that $g_{j}=g_{j}^{2}=g_{j}^{*} \neq 0, g_{j} \in\left[L_{1}, \cdots, L_{j}\right]$ and $g_{i} g_{j}=0$ for $i \neq j, i, j=1,2, \cdots$, $k-1$. We next show that $g_{k}$ may be defined with the corresponding properties.

Let $v=u_{k}-\sum_{j=1}^{k-1} u_{k} g_{j} . \quad$ Since $L_{k} \not \subset\left[L_{1}, \cdots, L_{k-1}\right], u_{k} \notin\left[L_{1}, \cdots, L_{k-1}\right]$ and thus $v \neq 0$. Since $L_{k}=R u_{k}$ is a minimal left ideal $u_{k} R u_{k}$ is a division ring with unit $u_{k}$. The propriety of the involution then yields $v v^{*} \neq 0$. Since $v v^{*} \in u_{k} R u_{k}$, there is an element $s \in u_{k} R u_{k}$ such that $s\left(v v^{*}\right)=\left(v v^{*}\right) s=u_{k}$. If we apply the involution to the prior relation $\left(v v^{*}\right) s^{*}=s^{*}\left(v v^{*}\right)=u_{k}$, and the uniqueness of inverses in a division ring yields $s=s^{*}$.

It is claimed that $g_{k}=v^{*} s v$ has the desired properties. Since $v g_{k} v^{*}=v v^{*} s v v^{*}=u_{k} v v^{*}=v v^{*} \neq 0$, it follows that $g_{k} \neq 0$. Clearly $g_{k}=g_{k}^{*}$ and $g_{k}^{2}=v^{*} s v v^{*} s v=v^{*} u_{k} s v=v^{*} s v=g_{k}$. If $i=1, \cdots, k-1$, $g_{i} v^{*}=g_{i}\left(u_{k}-\sum_{j=1}^{k-1} g_{j} u_{k}\right)=0$ by the inductive hypothesis, thus $g_{i} g_{k}=$ $g_{i} v^{*} s v=0$. By applying the involution we obtain $g_{k} g_{i}=0$. The induction is thus complete and we may suppose that $g_{1}, \cdots, g_{n}$ have been defined.

Clearly $\left[g_{1}\right]=\left[L_{1}\right]$. Suppose that for $1<k \leqq n,\left[g_{1}, \cdots, g_{k-1}\right]=$ $\left[L_{1}, \cdots, L_{k-1}\right]$. The defining property for $g_{k}$ yields $\left[g_{1}, \cdots, g_{k}\right] \subset$ $\left[L_{1}, \cdots, L_{k}\right]=\left[\left[g_{1}, \cdots, g_{k-1}\right], L_{k}\right]$. Thus $g_{k}=x_{1} g_{1}+\cdots+x_{k-1} g_{k-1}+x_{k} e_{1 k}$. Right multiplication of the last relation by $g_{k}$ shows that $x_{k} e_{1 k} \neq 0$. Since $L_{k}$ is a minimal left ideal, there is a $z \in R$ such that $z x_{k} e_{1 k}=e_{1 k}$. This may be expressed as $z\left[g_{k}-x_{1} g_{1} \cdots-x_{k-1} g_{k-1}\right]=e_{1 k}$. Thus $L_{k} \subset$ $\left[g_{1}, \cdots, g_{k}\right]$ and hence $\left[g_{1}, \cdots, g_{k}\right]=\left[L_{1}, \cdots, L_{k}\right]$ for $k=1, \cdots, n$. In particular $R=\left[g_{1}, \cdots, g_{n}\right]$.

The spaces $R g_{k}$ must be irreducible over $R$, otherwise we would have $R$ decomposed into sums of irreducible $R$-spaces of different lengths. Thus the ideals $R g_{k}$ are minimal. Furthermore if we denote the unit element of $R$ by $e$, we have $e=y_{1} g_{1}+\cdots+y_{n} g_{n}$. Right multiplication by $g_{j}$ shows that $g_{j}=y_{j} g_{j}$ and thus $e=g_{1}+\cdots+g_{n}$.

The form of an idempotent in $e_{11} R$ and $R e_{k k}, k=2, \cdots, n$, together with the fact that $\lambda e_{i j}=e_{i j} \lambda$ yields $\lambda u_{k}=u_{k} \lambda=u_{k} \lambda u_{k}, k=1, \cdots, n$ for any $\lambda \in D$. The inductive method of defining $g_{k}$ then permits one to 
deduce that $\lambda g_{k}=g_{k} \lambda=g_{k} \lambda g_{k}$. For suppose that $\lambda g_{j}=g_{j} \lambda$ for $j=1$, $\cdots, k-1$. From the way in which $v$ and $v^{*}$ were defined $\lambda v=v \lambda$ and $\lambda v^{*}=v^{*} \lambda$. Since $\lambda g_{k}=v^{*} \lambda s v=v^{*} u_{k} \lambda s u_{k} v$, and $g_{k} \lambda=v^{*} s \lambda v=v^{*} u_{k} s \lambda u_{k} v$, it is sufficient if we show that $u_{k} \lambda s u_{k}=u_{k} s \lambda u_{k}$ for all $\lambda \in D$. But $\left(u_{k} s \lambda u_{k}\right)\left(v v^{*}\right)=s v v^{*} \lambda=u_{k} \lambda=\lambda u_{k}=\lambda s v v^{*}=u_{k} \lambda s u_{k}\left(v v^{*}\right)$. Since $u_{k} R u_{k}$ is a division ring, $u_{k} s \lambda u_{k}=u_{k} \lambda s u_{k}$ as desired. Hence $\lambda g_{k}=g_{k} \lambda$ for all $\lambda \in D$ and $k=1, \cdots, n$.

Since $(0) \neq R g_{i} R$ is a two sided ideal of $R, R g_{i} R g_{k}=R g_{k} \neq(0)$, and thus $g_{i} R g_{k} \neq(0)$. Suppose $i<k$, and $g_{i} r g_{k} \neq 0$. Then, by the propriety of the involution, $0 \neq\left(g_{i} r g_{k}\right)\left(g_{i} r g_{k}\right)^{*}=g_{i} r g_{k} r^{*} g_{i}$. Since the left ideal $R g_{i}$ is minimal, $g_{i} R g_{i}$ is a division ring, and there exists $t \in R$ such that $\left(g_{i} t g_{i}\right)\left(g_{i} r g_{k} r^{*} g_{i}\right)=g_{i}$. If we take adjoints of the expressions in the preceding equation, we see that $g_{i} t g_{i}=g_{i} t^{*} g_{i}$. Let $g_{i k}=g_{i} t g_{i} r g_{k}$ and $g_{k i}=g_{k} r^{*} g_{i}$. Then $g_{i k} g_{k i}=g_{i}$, and consequently $\left(g_{i k} g_{k i}\right)\left(g_{i k} g_{k i}\right)=g_{i}$, so $0 \neq g_{k i} g_{i k} \in g_{k} R g_{k}$, which is a division ring. Also $g_{k i} g_{i k}$ is idempotent so $g_{k i} g_{i k}=g_{k}$. Finally if we define $g_{i i}=g_{i}$, we obtain a set of matrix units $\left\{g_{i j}\right\}$ for $R$ such that $g_{i i}^{*}=g_{i i}$. The form of the involution * on $R$ is then an immediate consequence of a theorem of Jacobson and Rickart [2].

We are now in a position in which we may discuss the continuity of involutions.

THEOREM 3. Let $D$ be a topological division ring such that any involution on $D$ is continuous. If $R$ is a total matric ring over $D$, then any proper ring involution on $R$ is continuous.

The result is immediate by virtue of the representation of the involution given in Theorem 2, together with the fact that convergence in $R$, when it is regarded as a finite dimensional vector space, involves [1] convergence of the coefficients of the representation in terms of a given basis.

We turn now to locally compact semi-simple rings which are either connected or compact. The first item needed concerns their topological algebraic structure.

Lemma. (a) A compact semi-simple ring is the topological direct sum of total matric algebras over finite fields.

(b) A locally compact connected semi-simple ring is the topological direct sum of a finite number of total matric rings over locally compact division rings.

Statement (a) is immediate from Theorem 16 of Kaplansky [4]. In the second statement, the semi-simplicity allows the use of Theorem 2 of Kaplansky [5], which shows that the ring is the direct sum of a semi-simple algebra over the reals with a unit and a totally disconnected ring. Since the decomposition is the Peirce decomposition relative to 
the algebra unit, it is easily seen that one has a topological direct sum. The connectedness then forces the second summand to be zero. The conclusion of the lemmas then follows from Theorem 10 of [5].

It might further be noted that the division rings involved must be connected. Consequently, since the only connected locally compact division rings are the reals, the complexes and the quaternions [3], [6], these are the only rings involved in the conclusion of $(b)$.

LEMMA 5. If ${ }^{*}$ is a proper involution on a direct sum of total matric rings over division rings, then each matric ring is invariant under *. Thus * restricted to an individual matric ring is a proper involution on that ring.

Let $R$ be the direct sum of rings $R_{j}$. Let $e^{\circ}$ be the unit of a summand $R^{\circ}$. Say $e^{\circ}=e_{1}+\cdots+e_{n}$ is the decomposition of $e^{\circ}$ in terms of the vector units of $R^{\circ}$. The right ideal $e_{i} R=e_{i} R^{\circ}$ is a minimal right ideal of $R$. Hence, by the theorem of Rickart used previously, there exists a unique idempotent $f_{k}$ in $e_{i} R$ such that $0 \neq f_{i}=f_{i}^{*}$. Thus $e_{i}=f_{i} e_{i}$ and $e_{i}^{*}=e_{i}^{*} f_{i}$. Consequently if $x \in R^{\circ}, x=e_{1} x+\cdots+e_{n} x=$ $f_{1} e_{1} x+\cdots+f_{n} e_{n} x$, and $x^{*}=x^{*} e_{1}^{*} f_{1}+\cdots+x^{*} e_{n}^{*} f_{n}$ is in $R^{\circ}$.

We are now in a position to establish the continuity of proper involutions on the class of semisimple rings under discussion.

THEOREM 6. If $R$ is a semi-simple locally compact ring which is either compact or connected then any proper involution * on $R$ is continuous.

In view of Lemmas 4 and 5 , it is sufficient to prove the continuity of ${ }^{*}$ on an individual matric ring. Thus the proof is complete for the compact ring. For the connected ring, all we need note is that the only involutions on the reals, complexes and quaternions are automatically continuous. Hence Theorem 3 applies and the proof is complete.

\section{REFERENCES}

1. N. Bourbaki, Eléments de mathématique, Livre $V$, Espaces vectoriels topologiques, Herman et Cie, Act. Sci. et Ind. 1189, 1229, Paris 1953, 1955.

2. N. Jacobson and C. E. Rickart, Homomorphisms of Jordan rings of self-adjoint elements, Trans. Amer. Math. Soc., 72 (1952), 310-322.

3. N. Jacobson and O. Taussky, Locally compact rings, Proc. Nat. Acad., Sci. U.S.A., 21 (1935), 106-108.

4. I. Kaplansky, Topological rings, Amer. J. Math., 69 (1947), 153-183.

5. L Locally compact rings, Amer. J. Math., 70 (1948), 447-459.

6. Y. Otobe, Note on locally compact simple rings, Proc. Imp. Acad. Tokyo, 20 (1944), 283.

7. C. E. Rickart, Representation of certain Bancach algebras, Duke Math. J., 18 (1951), $27-39$. 


\section{PACIFIC JOURNAL OF MATHEMATICS}

\section{EDITORS}

David GILbarg

Stanford University

Stanford, California

\section{F. H. BRowneLL}

University of Washington

Seattle 5, Washington

\section{A. L. Whiteman}

University of Southern California Los Angeles 7, California

\section{J. PAIGe}

University of California

Los Angeles 24, California

\section{ASSOCIATE EDITORS}
E. F. BECKENBACH
T. M. CHERRY
D. DERRY

\author{
E. HEWITT \\ A. HORN \\ L. NACHBIN
}

\author{
M. OHTSUKA \\ H. L. ROYDEN \\ M. M. SCHIFFER
}

E. SPANIER

E. G. STRAUS

F. WOLF

\section{SUPPORTING INSTITUTIONS}

\author{
UNIVERSITY OF BRITISH COLUMBIA \\ CALIFORNIA INSTITUTE OF TECHNOLOGY \\ UNIVERSITY OF CALIFORNIA \\ MONTANA STATE UNIVERSITY \\ UNIVERSITY OF NEVADA \\ NEW MEXICO STATE UNIVERSITY \\ OREGON STATE COLLEGE \\ UNIVERSITY OF OREGON \\ OSAKA UNIVERSITY \\ UNIVERSITY OF SOUTHERN CALIFORNIA
}

\author{
STANFORD UNIVERSITY \\ UNIVERSITY OF TOKYO \\ UNIVERSITY OF UTAH \\ WASHINGTON STATE COLLEGE \\ UNIVERSITY OF WASHINGTON \\ AMERICAN MATHEMATICAL SOCIETY \\ CALIFORNIA RESEARCH CORPORATION \\ HUGHES AIRCRAFT COMPANY \\ SPACE TECHNOLOGY LABORATORIES \\ NAVAL ORDNANCE TEST STATION
}

\footnotetext{
Mathematical papers intended for publication in the Pacific Journal of Mathematics should be typewritten (double spaced), and the author should keep a complete copy. Manuscripts may be sent to any one of the four editors. All other communications to the editors should be addressed to the managing editor, L. J. Paige at the University of California, Los Angeles 24, California.

50 reprints per author of each article are furnished free of charge; additional copies may be obtained at cost in multiples of 50 .
}

The Pacific Journal of Mathematics is published quarterly, in March, June, September, and December. The price per volume (4 numbers) is $\$ 12.00$; single issues, $\$ 3.50$. Back numbers are available. Special price to individual faculty members of supporting institutions and to individual members of the American Mathematical Society: $\$ 4.00$ per volume; single issues, $\$ 1.25$.

Subscriptions, orders for back numbers, and changes of address should be sent to Pacific Journal of Mathematics, 2120 Oxford Street, Berkeley 4, California.

Printed at Kokusai Bunken Insatsusha (International Academic Printing Co., Ltd.), No. 6, 2-chome, Fujimi-cho, Chiyoda-ku, Tokyo, Japan.

PUBLISHED BY PACIFIC JOURNAL OF MATHEMATICS, A NON-PROFIT CORPORATION

The Supporting Institutions listed above contribute to the cost of publication of this Journal, but they are not owners or publishers and have no responsibility for its content or policies. 


\section{Pacific Journal of Mathematics}

\section{Vol. 10, No. $4 \quad$ December, 1960}

M. Altman, An optimum cubically convergent iterative method of inverting a linear bounded operator in Hilbert space . . . . . . . . . . . . . . . . . . . . . . . . . . 1107

Nesmith Cornett Ankeny, Criterion for rth power residuacity ................. 1115

Julius Rubin Blum and David Lee Hanson, On invariant probability measures I . . . . . 1125

Frank Featherstone Bonsall, Positive operators compact in an auxiliary topology ..... 1131

Billy Joe Boyer, Summability of derived conjugate series . . . . . . . . . . . . . . . . 1139

Delmar L. Boyer, A note on a problem of Fuchs . . . . . . . . . . . . . . . . . 1147

Hans-Joachim Bremermann, The envelopes of holomorphy of tube domains in infinite

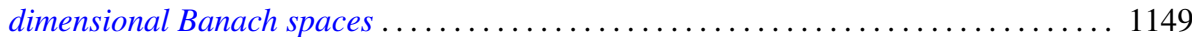

Andrew Michael Bruckner, Minimal superadditive extensions of superadditive

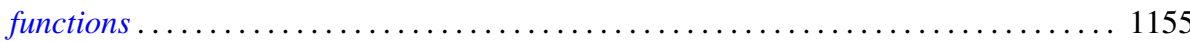

Billy Finney Bryant, On expansive homeomorphisms .................... 1163

Jean W. Butler, On complete and independent sets of operations in finite algebras . . . . . 1169

Lucien Le Cam, An approximation theorem for the Poisson binomial distribution ...... 1181

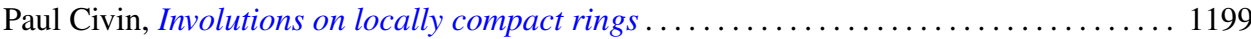

Earl A. Coddington, Normal extensions of formally normal operators . . . . . . . . . 1203

Jacob Feldman, Some classes of equivalent Gaussian processes on an interval ........ 1211

Shaul Foguel, Weak and strong convergence for Markov processes . . . . . . . . . . . 1221

Martin Fox, Some zero sum two-person games with moves in the unit interval ........ 1235

Robert Pertsch Gilbert, Singularities of three-dimensional harmonic functions . . . . . . . 1243

Branko Grünbaum, Partitions of mass-distributions and of convex bodies by

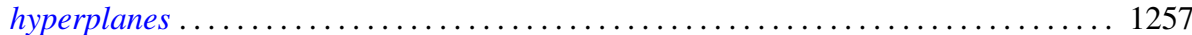

Sidney Morris Harmon, Regular covering surfaces of Riemann surfaces ........... 1263

Edwin Hewitt and Herbert S. Zuckerman, The multiplicative semigroup of integers

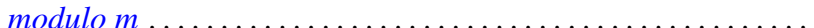

Paul Daniel Hill, Relation of a direct limit group to associated vector groups . ......... 1309

Calvin Virgil Holmes, Commutator groups of monomial groups . .

James Fredrik Jakobsen and W. R. Utz, The non-existence of expansive homeomorphisms

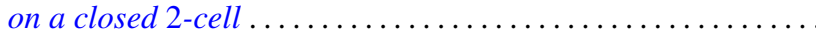

John William Jewett, Multiplication on classes of pseudo-analytic functions . . . . . . . 1323

Helmut Klingen, Analytic automorphisms of bounded symmetric complex domains . . . . 1327

Robert Jacob Koch, Ordered semigroups in partially ordered semigroups . . . . . . . . 1333

Marvin David Marcus and N. A. Khan, On a commutator result of Taussky and

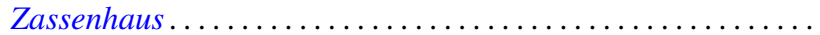

John Glen Marica and Steve Jerome Bryant, Unary algebras......

Edward Peter Merkes and W. T. Scott, On univalence of a continued fraction . . . . . . . 1361

Shu-Teh Chen Moy, Asymptotic properties of derivatives of stationary measures . . . . . 1371

John William Neuberger, Concerning boundary value problems . . . . . . . . . . . 1385

Edward C. Posner, Integral closure of differential rings . . . . . . . . . . . . . . . . . 1393

Marian Reichaw-Reichbach, Some theorems on mappings onto . . . . . . . . . . . . . 1397

Marvin Rosenblum and Harold Widom, Two extremal problems . . . . . . . . . . . . . . . . 1409

Morton Lincoln Slater and Herbert S. Wilf, A class of linear differential-difference

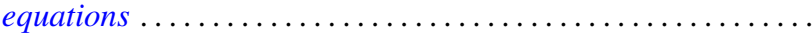

Charles Robson Storey, Jr., The structure of threads . . . . . . . . . . . . . . . . . . 1429

J. François Treves, An estimate for differential polynomials in $\partial / \partial z_{1},, \cdots, \partial / \partial z_{-} n \ldots \ldots 1447$

J. D. Weston, On the representation of operators by convolutions integrals . . . . . . . . 1453

James Victor Whittaker, Normal subgroups of some homeomorphism groups ......... 1469 